\title{
Resolving the Disk-Halo Degeneracy: A look at M74
}

\author{
S. Aniyan ${ }^{1}$, K. C. Freeman ${ }^{1}$, M. Arnaboldi ${ }^{2}$, O. Gerhard ${ }^{3}$, \\ L. Coccato ${ }^{2}$, K. Kuijken ${ }^{4}$ and M. Merrifield ${ }^{5}$ \\ ${ }^{1}$ Research School of Astronomy \& Astrophysics, Australian National University \\ email: suryashree.aniyan@anu.edu.au \\ ${ }^{2}$ European Southern Observatory, Garching \\ ${ }^{3}$ Max-Planck-Institut fur Extraterrestrische Physik, Garching \\ ${ }^{4}$ Leiden Observatory, Leiden University \\ ${ }^{5}$ School of Physics and Astronomy, University of Nottingham
}

\begin{abstract}
The decomposition of the $21 \mathrm{~cm}$ rotation curve of galaxies into contribution from the disk and dark halo depends on the adopted mass to light ratio $(\mathrm{M} / \mathrm{L})$ of the disk. Given the vertical velocity dispersion $\left(\sigma_{z}\right)$ of stars in the disk and its scale height $(h)$, the disk surface density and hence the M/L can be estimated. Earlier works have used this technique to conclude that galaxy disks are submaximal. Here we address an important conceptual problem: $\sigma_{z}$ and $h$ must pertain to the same population. Our analysis concludes that previous studies underestimate the disk surface mass density by $\sim 2$, sufficient to make a maximal disk for M74 appear like a submaximal disk.
\end{abstract}

Keywords. galaxies: kinematics and dynamics, galaxies: spiral, galaxies: halos

The disk-(dark) halo degeneracy in galaxies is still an important issue. The vertical stellar velocity dispersion $\sigma_{z}$ and the vertical disk scale height $h$ together give the surface density of the disk: $\Sigma=f \sigma_{z}^{2} / G h$, where $f$ is a geometric factor of order unity. $\Sigma$ and the surface brightness give the $\mathrm{M} / \mathrm{L}$ value of the disk. Previous work by Herrmann \& Ciardullo (2009) used planetary nebulae (PNe) as dynamical tracers in M74 and other disks to conclude that disks are mostly submaximal. Other works have used absorption line spectroscopy to conclude that disks are submaximal (see Bershady et al. 2011). However, a conceptual problem has so far been overlooked $-h$ is obtained from near-IR studies of edge-on galaxies and refer to the older kinematically hotter stars, while $\sigma_{z}$ is measured from face-on spirals in the optical bands and is representative of stars of all ages in the disk. To fix this problem, we need to measure the $\sigma_{z}$ for the old population.

We use PNe as tracers, observed with the Planetary Nebula Spectrograph on the William Herchel telescope, in the nearby spiral M74. We measure PNe velocities with typical errors $\leqslant 10 \mathrm{~km} / \mathrm{s}$. We are able to derive two independent velocity dispersion $\left(\sigma_{\mathrm{L} \text { os }}\right)$ values, for the kinematically hotter and colder stars. The hotter, old disk component has a $\sigma_{\mathrm{LOS}}$ that rises exponentially towards the center. The presence of the younger colder stars suggests that the previously determined surface mass density of the disk of M74 is underestimated by a factor $\sim 2$, consistent with our earlier study of the kinematics of K-giants in the solar neighbourhood (see Aniyan et al. 2016).

\section{References}

Herrmann, K. A. \& Ciardullo, R. 2009, ApJ, 705, 1686

Bershady, M. A., et al. 2011, ApJL, 739, L47

Aniyan, S., et al. 2016, MNRAS, 456, 1484 